\title{
ON ELEMENTARY AMENABLE GROUPS OF FINITE HIRSCH NUMBER
}

\author{
B. A. F. WEHRFRITZ
}

(Received 15 June 1992; revised 15 August 1992)

Communicated by H. Lausch

\begin{abstract}
We give an alternative short proof of a recent theorem of J.A. Hillman and P.A. Linnell that an elementary amenable group with finite Hirsch number has, modulo its locally finite radical, a soluble normal subgroup with index and derived length bounded only in terms of the Hirsch number of the group.
\end{abstract}

1991 Mathematics subject classification (Amer. Math. Soc.): $20 \mathrm{~F} 19$.

For any group $G$, let $\tau(G)$ denote its unique maximal locally finite normal subgroup. In [2] Hillman and Linnell prove that there are integer-valued functions $d(h)$ and $M(h)$ of $h$ only such that for any elementary amenable group $G$ with finite Hirsch number $h(G)=h$ there is a soluble normal subgroup of $G / \tau(G)$ with derived length at most $d(h)$ and index at most $M(h)$, the case where $h(G) \leq 3$ being dealt with in [1]. Here we offer an alternative short proof of this theorem, a proof that also makes explicit a little more of the structure of these groups. The phrase 'bounded by an integer-valued function of $n$ only' we shorten to ' $n$-bounded'.

(a) Let $G$ be a group with $\tau(G)=\langle 1\rangle$ and with a torsion-free abelian normal subgroup $A$ of finite rank $r$ such that $G / A$ is locally finite. Then $G$ has a torsion-free abelian normal subgroup with rank $r$ and $r$-bounded index.

Set $C=C_{G}(A)$. Then $C$ is centre by locally-finite, so $C^{\prime}$ is locally finite (Schur) and $C^{\prime}=\langle 1\rangle$. Then $C$ is torsion-free abelian, necessarily of rank $r$. Also $G / C$ is isomorphic to a locally finite subgroup of $G L(r, \mathbb{Q})$ and therefore has $r$-bounded order ( [6, 9.33 ii \& iii $]$ ).

(C) 1995 Australian Mathematical Society 0263-6115/95 \$A2.00+0.00 
(b) Let $T$ be a normal subgroup of finite order $m$ of a group $G$ such that $G / T$ is torsion-free abelian of rank $r<\infty$. Then $G$ has a torsion-free abelian normal subgroup of finite rank $r$ and index dividing $m^{2 r+1} \cdot m !$.

Set $C=C_{G}(T)$. Then $(G: C)$ divides $m$ !. Also $C$ is nilpotent of class at most 2 with $\left|C^{\prime}\right|$ dividing $m$. Hence $A=C^{m}$ is abelian, so $A^{m}$ is torsion-free abelian, necessarily of rank $r$. Also $\left(C: A^{m}\right)$ divides $m^{2 r+1}$.

Let $\mathfrak{X}_{h}$ denote the class of all groups $G$ with a series of finite length whose factors are locally finite or torsion-free abelian, such that the sum $h(G)$ of the ranks of the torsion-free abelian factors is at most $h$. Here $h(G)$ is an invariant of $G$. By (a minor extension of) a theorem of Mal'cev ( [4, Theorem 3]) the factor $G / \tau(G)$ of such a group $G$ has a poly torsion-free abelian, characteristic subgroup of finite index. Stronger still is the following.

(c) There is an integer-valued function $i(h)$ of $h$ only such that a group $G$ in $\mathfrak{X}_{h}$ has a characteristic subgroup $S$ with $(G: S) \leq i(h)$ and $S / \tau(S)$ poly torsion-free abelian of derived length at most $h$. The maximal soluble normal subgroup of $G / \tau(G)$ has derived length at most $h+i(h)$ and index at most $i(h)$.

We induct on $h$. Clearly we may assume that $\langle 1\rangle=\tau(G)<G$. Then $G$ has normal subgroups $G_{1} \leq H_{1}$ with $G / H_{1}$ locally finite and $H_{1} / G_{1}$ torsion-free abelian of rank $r \geq 1$. By (a) we may assume that $\left(G: H_{1}\right)$ is $r$-bounded. Apply induction to $G_{1} \in \mathfrak{X}_{h-r}$. There exists a characteristic subgroup $S_{1}$ of $G_{1}$ with $S_{1}$ poly torsion-free abelian of $(h-r)$-bounded index in $G_{1}$. Certainly $S_{1}$ is normal in $G$, so apply (b) to $H_{1} / S_{1}$. We obtain $T_{1}$ normal in $H_{1}$ with $T_{1}$ poly torsion-free abelian and $\left(H_{1}: T_{1}\right)$ $h$-bounded. Set $S=G^{\left(G: T_{1}\right)}$. Then $S$ is a characteristic poly torsion-free abelian subgroup of $G$ and $(G: S) \leq\left(G: T_{1}\right)^{h+1}$ is $h$-bounded. Trivially $S$ has derived length at most $h(S)=h(G) \leq h$.

For any group $G$ define the subgroups $G^{[i]}$ of $G$ for integers $i \geq 0$ by $G^{[0]}=G$, $G^{[1]} / G^{\prime}=\tau\left(G / G^{\prime}\right)$ and inductively $G^{[i+1]}=\left(G^{[i]}\right)^{[1]}$. Then $S^{[h]}$ in (c) is locally finite. If $H$ is a subgroup of $G$ then $H^{\prime} \leq G^{\prime}$, so $H^{[1]} \leq G^{[1]}$ and a simple induction yields that $H^{[i]} \leq G^{[i]}$ for each $i$. Also if $g \in G$ then $g \in G^{[1]}$ if and only if there exist elements $x_{i}$ and $y_{i}$ of $G$ and a positive integer $n$ such that $g^{n}=\prod\left[x_{i}, y_{i}\right]$. Thus $G^{[i]}=\bigcup_{X} X^{[i]}$, where $X$ ranges over the finitely generated subgroups of $G$.

(d) $\boldsymbol{X}_{h}$ is locally closed.

Let $G \in L \mathbb{X}_{h}$. Then (c) and the usual inverse limit argument (cf. [3, Section 1.K]) shows that $G$ has a normal subgroup $S$ of finite index at most $i(h)$ such that $S^{[h]}=\bigcup_{\text {f.g. } X \leq S} X^{[h]}$ is locally finite. If $h(G)>h$ then $h(X)>h$ for some finitely generated subgroup $X$ of $G$. Consequently $h(G) \leq h$ and $G \in \mathfrak{X}_{h}$.

(e) $\mathfrak{X}_{h} \mathfrak{X}_{k} \leq \mathfrak{X}_{h+k}$.

This is immediate from the definition of $\mathfrak{X}_{h}$. Clearly every group in $\mathfrak{X}_{h}$ is elementary amenable with Hirsch number (that is, Hirsch length in the sense of [2]) at most $h$ and so $\bigcup_{h \geq 0} \mathfrak{X}_{h}$ lies in the class of elementary amenable groups of finite Hirsch number. 
The converse follows easily from a trivial induction, using (d) and (e). Thus:-

(f) $\bigcup_{h \geq 0} \mathfrak{X}_{h}$ is the class of elementary amenable groups of finite Hirsch number and (c) gives the theorem of [2].

Let $G$ be an elementary amenable group with finite Hirsch number. Then in the notation of [5] the group $G / \tau(G)$ is a finite extension of a torsion-free $\$_{1}$-group and from this much follows. For example (c) can be strengthened as below (cf. $[5,10.33$, p.169]).

(g) There is an integer-valued function $j(h)$ of $h$ only such that a group $G$ in $\mathfrak{*}_{h}$ has characteristic subgroups $\tau(G) \leq N \leq M$ with $N / \tau(G)$ torsion-free nilpotent, $M / N$ free abelian of finite rank and $(G: M)$ at most $j(h)$.

For example, with $S$ as in (c), set $M=\tau(G) \cdot \bigcap_{i} C_{S}\left(S^{[i]} / S^{[i+1]}\right)$ and use Mal'cev's Theorem $([6,3.6])$.

\section{References}

[1] J. A. Hillman, 'Elementary amenable groups and 4-manifolds with Euler characterictic 0', J.Austral. Math. Soc. (Series A) 50 (1991), 160-170.

[2] J. A. Hillman and P. A. Linnell, 'Elementary amenable groups of finite Hirsch length are locally-finite by virtually-solvable', J. Austral. Math. Soc. (Series A) 52 (1992), 237-241.

[3] O. H. Kegel and B. A. F. Wehrfritz, Locally finite groups (North-Holland, Amsterdam, 1973).

[4] A. I. Mal'cev, 'On certain classes of infinite soluble groups', Mat. Sb. 28 (1951), 567-588 (in Russian) Amer. Math. Soc. Transl. Ser. 2 Vol. 2 (1956), 1-21.

[5] D. J. S. Robinson, Finiteness conditions and generalized soluble groups 2 (Springer, Berlin, 1972).

[6] B. A. F. Wehrfritz, Infinite linear groups (Springer, Berlin, 1973).

School of Mathematical Sciences

Queen Mary \& Westfield College

Mile End Road

London E1 4NS

England 